\title{
PDE5 expression in human thyroid tumors and effects of PDE5 inhibitors on growth and migration of cancer cells
}

\author{
Marialuisa Sponziello $^{1}$ - Antonella Verrienti ${ }^{1}$. Francesca Rosignolo ${ }^{1} \cdot$ \\ Roberta Francesca De Rose $^{2}$ - Valeria Pecce ${ }^{1}$. Valentina Maggisano ${ }^{2}$. \\ Cosimo Durante ${ }^{1} \cdot$ Stefania Bulotta $^{2} \cdot$ Giuseppe Damante $^{3} \cdot$ Laura Giacomelli $^{4}$. \\ Cira Rosaria Tiziana Di Gioia ${ }^{5} \cdot$ Sebastiano Filetti $^{1} \cdot$ Diego Russo $^{2}$. \\ Marilena Celano ${ }^{2}$
}

Received: 12 January 2015 / Accepted: 25 March 2015

(C) Springer Science+Business Media New York 2015

\begin{abstract}
Recent studies have revealed in normal thyroid tissue the presence of the transcript of several phosphodiesterases (PDEs), enzymes responsible for the hydrolysis of cyclic nucleotides. In this work, we analyzed the expression of PDE5 in a series of human papillary thyroid carcinomas (PTCs) presenting or not BRAF V600E mutation and classified according to ATA risk criteria. Furthermore, we tested the effects of two PDE5 inhibitors (sildenafil, tadalafil) against human thyroid cancer cells. PDE5 gene and protein expression were analyzed in two different cohorts of PTCs by real-time PCR using a TaqMan microfluid card system and Western blot. MTT and migration assay were used to evaluate the effects of PDE5 inhibitors on proliferation and migration of TPC-1, BCPAP, and $8505 \mathrm{C}$ cells. In a first series of 36 PTCs, we found higher expression levels of $P D E 5 A$ in tumors versus non-tumor (normal) tissues. PTCs with $B R A F$ mutation showed higher
\end{abstract}

Marialuisa Sponziello and Antonella Verrienti have contributed equally to this work.

Diego Russo

d.russo@unicz.it

1 Department of Internal Medicine and Medical Specialties, University of Rome "Sapienza", 00161 Rome, Italy

2 Department of Health Sciences, University of Catanzaro "Magna Graecia", Campus "S. Venuta", Viale Europa, 88100 Catanzaro, Italy

3 Institute of Medical Genetics, University Hospital "S. Maria della Misericordia”, 33100 Udine, Italy

4 Department of Surgical Sciences, University of Rome "Sapienza", 00161 Rome, Italy

5 Department of Radiological, Oncological and Pathological Sciences, University of Rome "Sapienza", 00161 Rome, Italy levels of mRNA compared with those without mutation. No significant differences were detected between subgroups with low and intermediate ATA risk. Upregulation of PDE5 was also detected in tumor tissue proteins. Similar results were obtained analyzing the second cohort of 50 PTCs. Moreover, all tumor tissues with high PDE5 levels showed reduction of Thyroglobulin, TSH receptor, Thyroperoxidase, and NIS transcripts. In thyroid cancer cells in vitro, sildenafil and tadalafil determined a reduction of proliferation and cellular migration. Our findings demonstrate for the first time an overexpression of PDE5 in PTCs, and the ability of PDE5 inhibitors to block the proliferation of thyroid cancer cells in culture, therefore, suggesting that specific inhibition of PDE5 may be proposed for the treatment of these tumors.

Keywords Papillary thyroid carcinoma . Phosphodiesterases · BRAF - Thyroid cancer cells

\section{Introduction}

An increase of thyroid carcinomas, and in particular the papillary histotype, has been registered in the last decade $[1,2]$. Together with the well-differentiated forms, which are efficaciously managed by the treatment with surgery and radioiodine, an increased prevalence was reported also for the less differentiated carcinomas [1, 3]. These tumors are unable to concentrate this radioisotope and do not respond to the standard treatment or develop radio resistance $[4,5]$. These unresponsive tumors are characterized by an altered phenotype, often including the reduction/loss of expression of sodium/iodide symporter (NIS) and thyroperoxidase (TPO), owing to the oncogenic activation of intracellular signal transduction pathways which control 
cell growth and differentiation [6-9]. Study of the regulation of intracellular messengers, therefore, may help to identify molecular targets for novel therapeutic approaches.

Alterations of intracellular cyclic AMP (cAMP) levels deriving by mutations in the TSH receptor $(T S H-R)$ gene have been extensively investigated, and it is well established their pathogenic role in several thyroid diseases, including hyperfuncting adenomas (gain-of-function mutations) as well as forms of hypothyroidism (inactivating TSHR mutations) [10-12]. Less defined is the role of cGMP in thyroid cells $[13,14]$. Intracellular levels of the cyclic nucleotides are modulated by phosphodiesterases (PDEs), enzymes able to hydrolyze both cAMP and cGMP, and therefore acting as physiological regulators of all the processes mediated by such second messengers [15]. Normal thyroid tissues express subtypes PDE4, PDE5, PDE7, and PDE8 [16]. Moreover, expression of PDE4, able to hydrolyze the cAMP, was also reported to play a regulatory role in toxic adenomas carrying mutations in the $T S H-R$ gene [17]. In contrast, there are no data on the expression of PDE5 (responsible for hydrolysis of cGMP) in thyroid cancer cells.

In this study, we have analyzed the expression of PDE5 in surgical specimens of two series of human papillary thyroid tumor tissues ( $n=86$, in total), measuring both gene and, when available, protein expression levels, and comparing its expression with those of some thyroidspecific genes. The relationship with $B R A F$ mutational status and other biological features determining clinicpathologic features predicting the risk assessment (low or intermediate category) of recurrence was also investigated.

To verify the possibility to target the PDE5 for therapeutic purpose, we also tested the effects of two PDE5 inhibitors on the growth and migration properties of three human thyroid cell lines derived from papillary (TPC-1 and BCPAP cells) and anaplastic thyroid carcinoma (8505C cells).

\section{Materials and methods}

\section{Collection of thyroid tissues}

Tumor thyroid tissues were collected from patients subjected to thyroidectomy for PTC at the Sapienza's University Hospital of Rome, Policlinico Umberto I. Two independent cohorts were studied. An exploratory cohort $(n=36)$ was used for generating the hypothesis, a confirmatory cohort $(n=50)$ to test it. For each patient, a sample of thyroid tumor tissue and, when available, also contralateral normal tissue was collected, snap-frozen, and stored in liquid nitrogen. All 86 cases were risk-stratified (low or intermediate risk category) on the basis of clinical and pathological features in accordance with the 2009
American Thyroid Association (ATA) initial risk stratification system guidelines [18], and the presence or not of somatic BRAF V600E mutation detected by direct sequencing was performed in cDNA samples of tumor tissues as described previously [19]. All Patients' tumors information (exploratory and confirmatory cohorts combined) are reported in Table 1. All human tissue samples used in the study were collected with full Patients' informed written consent and approval from the Policlinico Umberto I Ethical Committee of Rome.

\section{Extraction of RNA and gene expression studies}

Total RNA was isolated using TRIzol reagent (Life Technologies, Foster City, CA, USA) following the manufacturer's recommended protocol and quantified with a NanoDrop Spectrophotometer (Thermo Fisher Scientific, Inc., Waltham, MA, USA). cDNAs were obtained following the protocol provided with the High Capacity cDNA

Table 1 Characteristics of the study population at the time of primary surgery

\begin{tabular}{|c|c|}
\hline Characteristic & Study cohort $(n=86)$ \\
\hline \multicolumn{2}{|l|}{$\operatorname{Sex}(n$, rate $)$} \\
\hline Male & $28(32.6)$ \\
\hline Female & $58(67.4)$ \\
\hline Age at diagnosis, years (median, range) & $44.5(18-81)$ \\
\hline \multicolumn{2}{|l|}{ Histological variant $(n$, rate) } \\
\hline PTC-cl & $72(83.7)$ \\
\hline PTC-fv & $12(14)$ \\
\hline PTC-other & $2(2.3)$ \\
\hline Tumor size, mm (median, range) & $12(3-38)$ \\
\hline \multicolumn{2}{|l|}{ Tumor foci $(n$, rate $)$} \\
\hline Unifocal & $69(80.2)$ \\
\hline Multifocal & $17(19.8)$ \\
\hline \multicolumn{2}{|l|}{ Extrathyroidal extension ( $n$, rate) } \\
\hline No & $52(60.5)$ \\
\hline Minor & $34(39.5)$ \\
\hline \multicolumn{2}{|l|}{ Lymph node metastases ( $n$, rate) } \\
\hline No & $56(65.1)$ \\
\hline Yes & $30(34.9)$ \\
\hline \multicolumn{2}{|l|}{$\operatorname{ATA} \operatorname{Risk}(n, \text { rate })^{\mathrm{a}}$} \\
\hline Low & $37(43)$ \\
\hline Intermediate & $49(57)$ \\
\hline \multicolumn{2}{|l|}{ BRAF mutational status ( $n$, rate) } \\
\hline wt & $30(34.8)$ \\
\hline V600E & $55(64)$ \\
\hline V600_K601delinsE & $1(1.2)$ \\
\hline
\end{tabular}

ATA American Thyroid Association, PTC-cl classical variant, $P T C-f v$ follicular variant, $w t$ wild type

a 2009 American Thyroid Association risk stratification staging system 
Reverse Transcription kit (Life Technologies). To evaluate the expression of PDE5A, NIS, TPO, Thyroglobulin (Tg), and $T S H-R$ genes in thyroid normal and tumor tissues, realtime PCR was performed using custom Taqman Low Density Arrays (TLDA, Life Technologies). Specific predesigned assays were performed to configure each TLDA (TaqMan Gene Expression Assays, Life Technologies) through one replicate for biological sample. Four housekeeping genes (Beta-actin, Glyceraldehyde-3-Phosphate Dehydrogenase, Hypoxanthine Phosphoribosyltransferase 1, and Beta-2 microglobulin) were carried out to normalize RNA expression levels [20]. $C_{\mathrm{T}}$ values were calculated through the SDS software (version 2.4) and used to calculate relative quantification (RQ) with RQ Manager software (Life Technologies). In detail, beta-actin was chosen as endogenous control due to its lower variation among samples, and final results were expressed as relative expression normalized to a calibrator sample.

\section{Protein extraction and western blot analysis}

Extraction of total proteins was performed as previously described [21]. $20 \mu \mathrm{g}$ of proteins was run on a 9 or $12 \%$ SDS-PAGE gel, transferred to PVDF membrane $(2 \mathrm{~h}$ at $225 \mathrm{~mA}$ ) (VWR, Milan, Italy), blocked with TTBS/milk (TBS, $1 \%$ Tween 20 and $5 \%$ non-fat dry milk) and incubated overnight with affinity-purified anti-PDE5 (Santa Cruz, Milan, Italy), diluted 1:500 for tissues and 1:100 for cells and anti- $\beta$-actin antibodies (Sigma Aldrich, Segrate, Milan, Italy) (used as internal control) diluted 1:10,000. The membranes were washed in TTBS and incubated with horseradish peroxidase-conjugated anti-rabbit or antimouse antibody (Transduction Laboratories, Lexington, KY, USA) diluted $1: 10,000$ or $1: 20,000$ in TTBS/milk. The protein was visualized by chemiluminescence using the Western blot detection system ECL (VWR) and Western lightning Plus-ECL (Perkin Elmer, Milan, Italy). Quantification was achieved by densitometric scanning.

\section{Thyroid cancer cell lines}

For in vitro experiment, we used human papillary (BCPAP and TPC-1, provided by Prof. A. Fusco) and anaplastic (8505C provided by Dr. C. Nucera) thyroid carcinoma cell lines, confirmed as of thyroid cancer origin and hosting the RET-PTC (TPC-1) or BRAF (BCPAP and 8505C) genotypic alteration, respectively [22]. The cells were cultured as previously described $[23,24]$.

\section{MTT assay}

TPC-1, BCPAP, and 8505C cells were seeded in 96-well plates at a density of $3 \times 10^{3}$ (TPC-1 and $8505 \mathrm{C}$ ) or
$5 \times 10^{3}$ (BCPAP). Proliferation was assessed by MTT [25]. After $24 \mathrm{~h}$, growth medium was replaced by fresh normal medium supplemented with sildenafil and tadalafil (Aurogene, Roma, Italy) $(1,10,100 \mu \mathrm{M})$, and cells were incubated for 24 and $48 \mathrm{~h}$. Then, the solubilized product was quantified with a microplate spectrophotometer (Multiskan MS 6.0, Labsystems) at a wavelength of $540 \mathrm{~nm}$ and a reference wavelength of $690 \mathrm{~nm}$. Results are expressed as percentages over untreated cultures (control).

\section{Migration assay}

Cell migration assay was performed using Transwell inserts with $8 \mu \mathrm{m}$ pore (Constar; Euroclone, Milan, Italy), as previously described [26]. Briefly, cells were treated with sildenafil $(0.01,0.1,1$, and $10 \mu \mathrm{M})$ and tadalafil $(0.01,0.1$, 1 , and $10 \mu \mathrm{M}$ ) for $24 \mathrm{~h}$, trypsinized, and centrifuged (1200 rpm for $5 \mathrm{~min}$ ). After suspension in medium without serum, the pellet was centrifuged (1200 rpm for $5 \mathrm{~min}$ ) and suspended in medium containing $1 \%$ BSA. Then, the cells were plated in the upper chamber of filters at a density of $50 \times 10^{3}$ cells (in $100 \mu \mathrm{L}$ ) while in the inferior chamber were added $600 \mu \mathrm{L}$ of medium containing $10 \%$ FBS. After $6 \mathrm{~h}$ of incubation, cells not migrated were removed from the upper surface of the filters with cotton swabs, while the filters were fixed and stained with Diff-Quick. Migrated cells were counted in five random fields using an eyepiece equipped with a counting grid.

\section{Statistical analysis}

The results are expressed as mean \pm standard deviation (SD). For cell proliferation and cell migration assay was adopted the one-way ANOVA followed by the TukeyKramer multiple comparisons test using GraphPad Prism version 5.0 statistical software (GraphPad Software Inc., San Diego, CA, USA). Analysis of mRNA levels was performed by Mann-Whitney test. $p$ values lower than 0.05 were considered statistically significant.

\section{Results}

\section{Gene and protein expression of PDE5 and clinical- pathological features of PTCs}

We first examined by TLDA the expression of PDE5A in a series of 36 tissues from patients with sporadic PTC (exploratory cohort). These tumors, according to ATA risk classification system, included 17 with a low degree of risk and 19 with intermediate risk. Among them, 7 and 14 carry the BRAF V600E mutation, respectively (Table 1). 
Fig. 1 mRNA levels of PDE5A in PTCs. Expression levels of PDE5A in two series of thyroid tumor tissues [exploratory group, $n=36$ (a); confirmatory group, $n=50(\mathbf{b})$ ] compared to pool of normal tissues. In both series, analysis of subgroups (PTCs with BRAF V600E vs. $B R A F$ wild type, and PTC classified as intermediate ATA risk vs. low risk) is reported. Data represent the mean $\pm \mathrm{SD}$. $p$ value was obtained by MannWhitney test. $* p<0.01$, $* * * p<0.0001, n s$ not significant
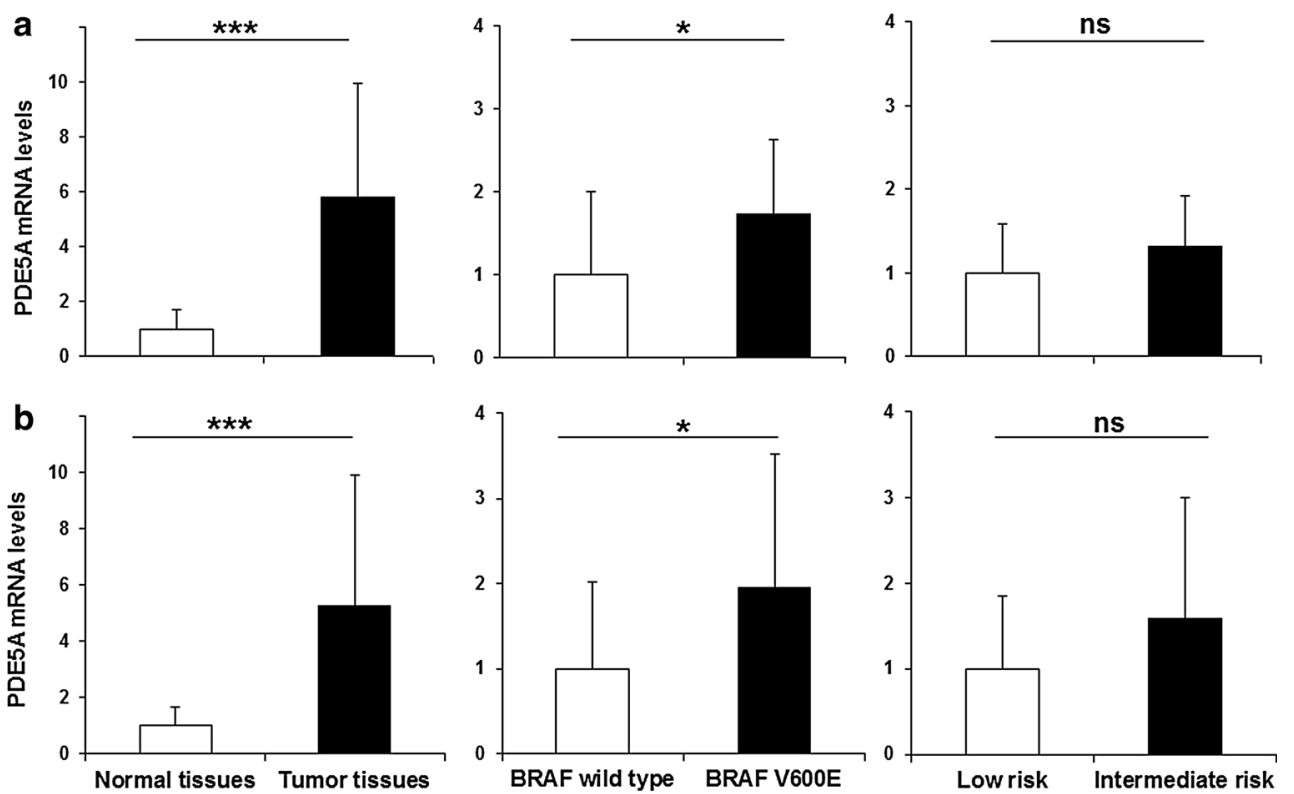

Expression levels of PDE5A resulted significantly higher in the tumor tissues compared to a pool of nontumor (normal) tissues and also in $B R A F \mathrm{~V} 600 \mathrm{E}$, when compared with $B R A F$ wild-type PTCs $(p<0.0001$ and $p<0.01$, respectively). No differences were observed between low risk and intermediate risk PTCs (Fig. 1a). This analysis was replicated in a wider and independent cohort of patients with sporadic PTCs $(n=50)$, and results were confirmed (Fig. 1b).

Data of PDE5A transcript levels were then compared with those of the thyroid-specific genes NIS, TPO, Tg, and $T S H-R$. As shown in Fig. 2, while almost all tumor samples had higher PDE5A expression levels as compared to normal samples, they displayed significantly lower mRNA levels of thyroid-specific genes (Fig. 2).

Subsequently, Western blot analysis performed in protein extracts of a series of PTCs showed higher level of protein expression in tumor tissues compared to normal counterpart (Fig. 3), confirming the finding obtained with mRNA analysis.

\section{Effects of sildenafil and tadalafil on proliferation and migration of thyroid cancer cells}

To evaluate the effects of PDE5 inhibition on thyroid tumor cells, we examined viability and migration properties of BCPAP, TPC-1 (from PTC), and 8505C (from ATC) cells incubated with sildenafil and tadalafil, two different specific inhibitors of PDE5. All cell lines showed detectable levels of PDE5 (Fig. 4). As shown in Fig. 5, treatment with $1,10,100 \mu \mathrm{M}$ of sildenafil or tadalafil for 24 and $48 \mathrm{~h}$ reduced the proliferation of all three cell lines with similar trend. In particular, the strongest inhibiting effect (about $50 \%$ vs. control) was observed using the $100 \mu \mathrm{M}$ concentration of tadalafil in all cell lines ( $p<0.05, p<0.01, p<0.001$ vs. control). At the same concentration, minor but significant effect was exerted by sildenafil on BCPAP and 8505C cells after $48 \mathrm{~h}(p<0.05$, $p<0.01$ vs. control).

Next, effects of PDE5 inhibitors on cell migration were evaluated. As shown in Fig. 6, in BCPAP and 8505C cell lines, a reduction of the migration was observed at $0.1,1$, and $10 \mu \mathrm{M}(8505 \mathrm{C}$ and BCPAP cells $\sim 50 \%$ vs. untreated cells; $p<0.001$ and 0.05 , respectively).

Interestingly and in accordance with our previous in vivo data on $B R A F$ mutated tumors, the cells carrying the BRAF V600E mutation (8505C and BCPAP) showed a better response to PDE5 inhibition in both anti-proliferative and anti-migration action of both inhibitors.

\section{Discussion}

Novel approaches are currently under investigation for the management of those differentiated thyroid cancers refractory to radioiodine therapy. A major alteration detected in such thyroid cancer cells regards the machinery responsible for iodide uptake and concentration, primary involving the sodium/iodide symporter, caused by genetic and epigenetic abnormalities especially detected in metastatic lesions [27-31]. Almost all the known genetic alterations occur in elements of signal transduction pathways controlling both cell growth and differentiation. In PTCs, activating BRAF mutation represents the most frequent genetic alteration and is also considered as a marker of aggressiveness [32-35]. 
Fig. 2 PDE5 and thyroidspecific genes mRNA levels in PTCs. mRNA levels of PDE5A (a), NIS (b), Tg (c), TPO (d), and $T S H-R(\mathbf{e})$, measured by real-time RT-PCR, are reported for each tumor. Results are expressed as fold of increase/ decrease $(\mathrm{RQ} \pm \mathrm{SD})$ relative to mean of normal thyroid tissues, considered arbitrarily as $1 . R Q$ relative quantification, $S D$ standard deviation

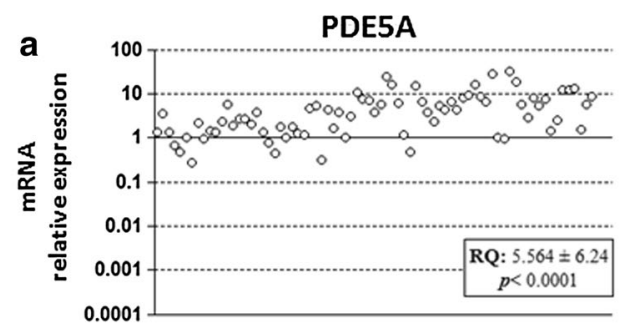

b
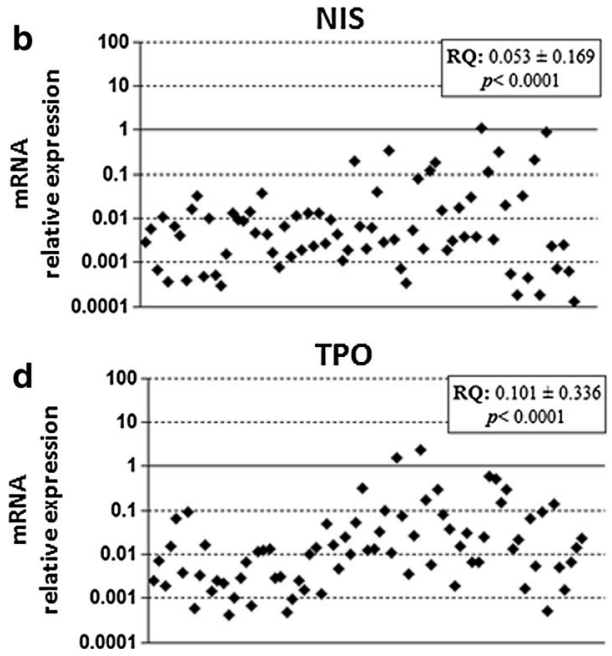

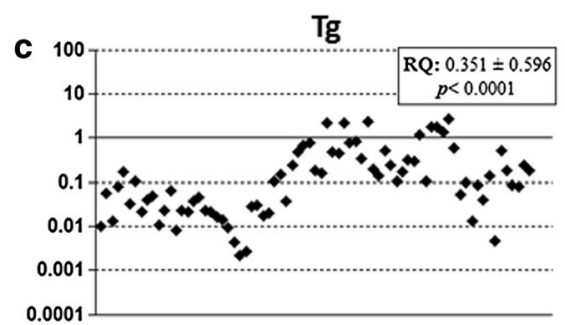

TSH-R

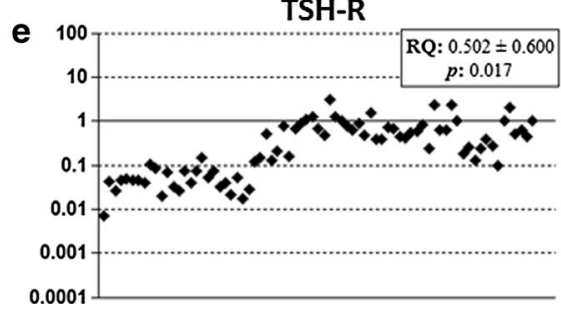

\section{BRAF V600E PTCs}

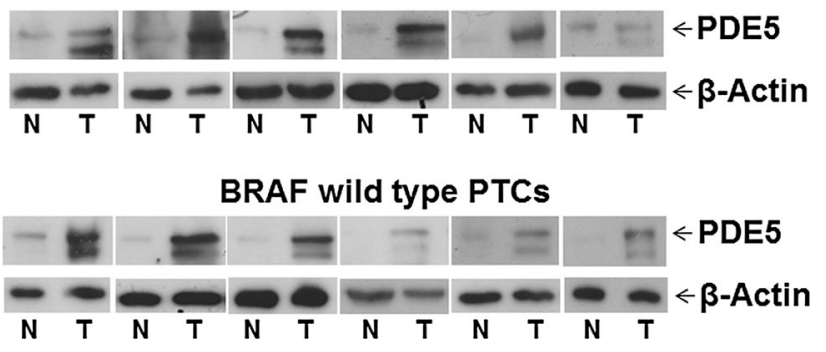

Fig. 3 Expression of PDE5 protein in PTCs. A couple of bands of approximately 95 and $100 \mathrm{kDa}$, corresponding to human PDE5 protein, was detected by Western blot analysis in the total protein extracts of normal $(\mathrm{N})$ and tumor $(\mathrm{T})$ tissues, with $B R A F$ wild type or carrying the V600E mutation. Data shown are relative to a representative group of samples

At variance with cAMP and its pathway, widely investigated as the main mediator of the TSH effects on thyroid cells and involved in several thyroid diseases [10], the role of the regulation of cGMP, mainly exerted by PDEs, in thyroid cancer has never been investigated. PDEs may be considered a key therapeutic target, and quantitative RTPCR analysis has revealed the mRNA expression profile of all PDE isoenzymes in human brain and peripheral tissues and in normal thyroid gland, which expressed the transcripts of PDE4, PDE7, PDE8, and PDE5 [16]. The latter enzyme acts specifically hydrolyzing the cGMP and is encoded by one gene, PDE5A, with three variants:
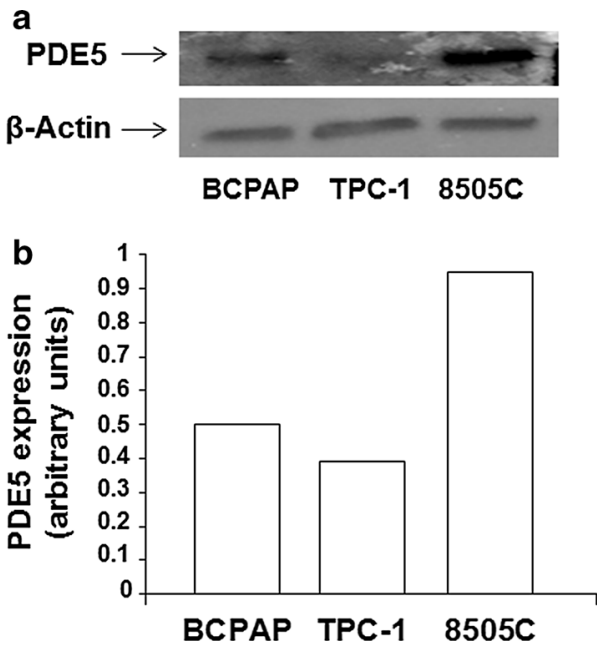

Fig. 4 PDE5 espression in thyroid cancer cells. PDE5 was detectable in BCPAP, TPC-1, and 8505C cells. Western blot analysis representative of three different experiments (a). Values were obtained from densitometric scanning bands from Western blot; the amounts of $\beta$ actin were evaluated for normalization (b)

PDE5A1 ( 100 kDa), PDE5A2 ( 95 kDa), and PDE5A3 ( $\sim 95 \mathrm{kDa})[36]$.

Our results show for the first time an increased expression of PDE5, both at mRNA and protein levels, in a total of 86 PTCs belonging to two independent cohorts. Increased transcript of PDE5A was also associated with decrease of the transcript of some differentiation markers as 

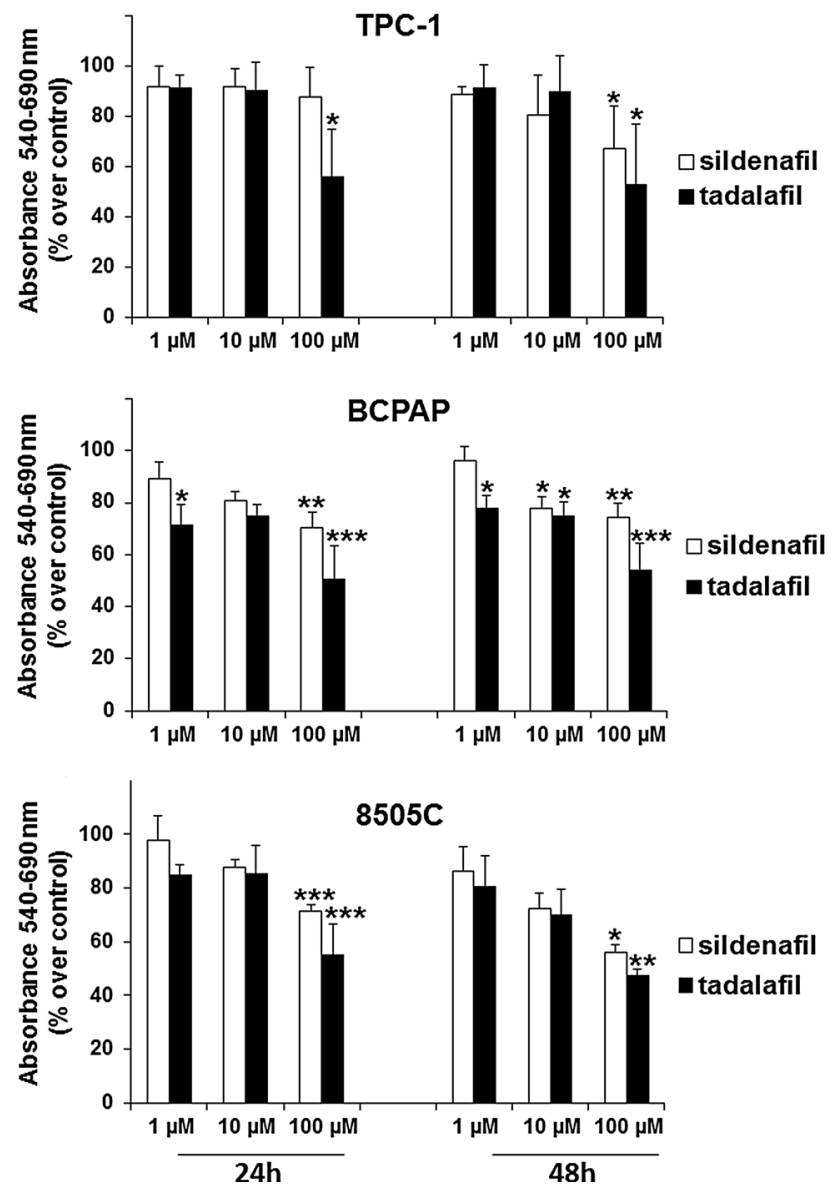

Fig. 5 Effects of sildenafil and tadalafil on cell viability. MTT assay was performed to analyze the effects of sildenafil and tadalafil at 1 , 10 , and $100 \mu \mathrm{M}$ after 24 and $48 \mathrm{~h}$ incubation on TPC-1, BCPAP, and $8505 \mathrm{C}$ cells. Results are expressed as mean $\pm \mathrm{SD}$ from at least three independent experiments performed in eigthplicate. ${ }^{*} p<0.05$ vs. control; **p $<0.01 ; * * * p<0.001$ vs. control

$T S H-R, T g, T P O$, and NIS, all implicated in intra-thyroidal iodine metabolism and thyroid hormone synthesis. Moreover, by analyzing the clinicobiological chart of the patients, we noted presence of higher expression levels of PDE in PTCs with BRAF V600E mutation. However, increase of PDE5A mRNA levels cannot be associated with the aggressiveness of the tumors, since no differences were observed comparing the subgroups classified according to the 2009 ATA criteria risk stratification system as low and intermediate risk. Interestingly, in a parallel study focusing on genome abnormality, amplification of PDE5A gene resulted a frequent lesion detected in PTCs, independent from their aggressiveness (unpublished observation).

The present findings prompted us to test the effects of PDE5 inhibition in thyroid cancer cells. We considered as bona fide results those determined in a concordant way by two different PDE5 inhibitors, sildenafil and tadalafil, currently approved for use in male erectile dysfunction and
TPC-1
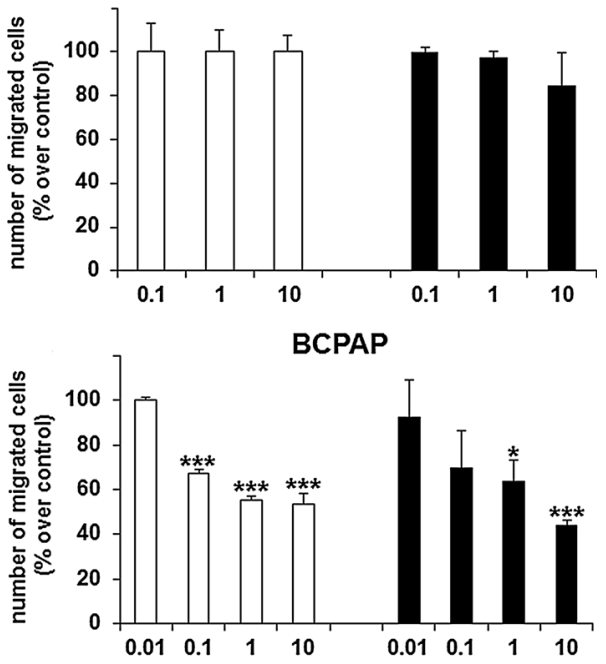

$8505 \mathrm{C}$

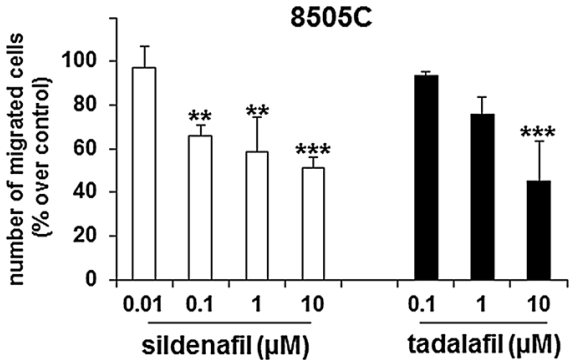

Fig. 6 Effect of sildenafil and tadalafil on cell migration. Migration through gelatine coated filters was analyzed after treatment with sildenafil or tadalafil for $24 \mathrm{~h}$. Results are mean \pm SD of three independent experiments, and statistical analysis was performed using the Tukey-Kramer multiple comparisons test. $* p<0.05$ vs. control; $* * p<0.01$ vs. control; ***p $<0.001$ vs. control

in pulmonary hypertension [37-40]. Moreover, there is considerable excitement regarding the potential use of PDE5 inhibitors as therapeutics for cardiovascular diseases, including hypertension, ischaemic cardiomyopathy, cardiac failure, stroke, peripheral myopathy, and cardiac dysfunction in Duchenne muscular dystrophy, as well as insulin resistance, type 2 diabetes, and metabolic syndrome, and several clinical trials are in progress [41]. Various PDE1 to PDE5 selective inhibitors have also been reported to inhibit growth and induce apoptosis in many different cancer cell lines, suggesting a potential role for this family of drugs also as antineoplastic agents [41].

We found a significant inhibitory action of both molecules on the growth of three human cancer cell lines. At lower doses, sildenafil and tadalafil were also able to reduce the capacity of the tumor cells to migrate, a fundamental step for the metastatic spread of the neoplastic disease. Also, the effects of sildenafil and tadalafil were stronger in the cell lines (BCPAP and 8505C) carrying the $B R A F$ mutation. Together with our in vivo results, higher 
expression of PDE5 in BRAF mutated tumors, this finding would suggest that these PTCs could represent a preferential target for the action of PDE5 inhibitors.

In conclusion, our finding demonstrate for the first time an overexpression of PDE5 in PTCs and the ability of PDE5 inhibitors to block the proliferation of thyroid cancer cells in culture. Further studies in experimental models in vivo will clarify whether specific inhibition of PDE5 may be proposed for the treatment of these tumors.

Acknowledgments This work was supported by Fondazione Umberto Di Mario.

Funding This work is funded by grant to MC (MIUR: Grant RBFR12FI27_003).

Conflict of interest The authors declare that they have no conflict of interest.

\section{References}

1. B. Aschebrook-Kilfoy, R.B. Schechter, Y.C. Shih, E.L. Kaplan, B.C. Chiu, P. Angelos, R.H. Grogan, The clinical and economic burden of a sustained increase in thyroid cancer incidence. Cancer Epidemiol. Biomark. Prev. 22(7), 1252-1259 (2013)

2. C.K. Jung, M.P. Little, J.H. Lubin, A.V. Brenner, S.A. Wells Jr, A.J. Sigurdson, Y.E. Nikiforov, The increase in thyroid cancer incidence during the last four decades is accompanied by a high frequency of BRAF mutations and a sharp increase in RAS mutations. J. Clin. Endocrinol. Metab. 99(2), E276-E285 (2014)

3. H.S. Kazaure, S.A. Roman, J.A. Sosa, Aggressive variants of papillary thyroid cancer: incidence, characteristics and predictors of survival among 43,738 patients. Ann. Surg. Oncol. 19(6), 1874-1880 (2012)

4. M. Schlumberger, L. Lacroix, D. Russo, S. Filetti, J.M. Bidart, Defects in iodide metabolism in thyroid cancer and implications for the follow-up and treatment of patients. Nat. Clin. Pract. Endocrinol. Metab. 3(3), 260-269 (2007)

5. C. Qin, W. Cau, Y. Zhang, F.P. Mghanga, X. Lan, Z. Gao, R. An, Correlation of clinicopathological features and expression of molecular markers with prognosis after ${ }^{131}$ I treatment of differentiated thyroid carcinoma. Clin. Nucl. Med. 37(3), e40-e46 (2012)

6. F. Trapasso, R. Iuliano, E. Chiefari, F. Arturi, A. Stella, S. Filetti, A. Fusco, D. Russo, Iodide symporter gene expression in normal and transformed rat thyroid cells. Eur. J. Endocrinol. 140, 447-451 (1999)

7. F. Arturi, D. Russo, J.M. Bidart, D. Scarpelli, M. Schlumberger, S. Filetti, Expression pattern of the pendrin and sodium/iodide symporter (NIS) gene in human thyroid carcinoma cell lines and human thyroid tumors. Eur. J. Endocrinol. 145, 129-135 (2001)

8. P. Soares, J. Lima, A. Preto, P. Castro, J. Vinagre, R. Celestino, J.P. Couto, H. Prazeres, C. Eloy, V. Máximo, M. SobrinhoSimões, Genetic alterations in poorly differentiated and undifferentiated thyroid carcinomas. Curr. Genomics 12, 609-617 (2011)

9. M. Xing, Molecular pathogenesis and mechanisms of thyroid cancer. Nat. Rev. Cancer 13(3), 184-199 (2013)

10. D. Russo, F. Arturi, E. Chiefari, S. Filetti, Molecular insights into TSH receptor abnormality and thyroid disease. J. Endocrinol. Invest. 20, 36-47 (1997)
11. D. Russo, C. Betterle, F. Arturi, E. Chiefari, M.E. Girelli, S. Filetti, A novel mutation in the Thyrotropin receptor gene causing loss of TSH binding but constitutive receptor activation in a family with resistance to TSH. J. Clin. Endocrinol. Metab. 85, 4238-4242 (2000)

12. T.F. Davies, T. Ando, R.Y. Lin, Y. Tomer, R. Latif, Thyrotropin receptor-associated diseases: from adenomata to Graves disease. J. Clin. Invest. 115(8), 1972-1983 (2005)

13. J. Van Sande, J. Mockel, J.M. Boeynaems, P. Dor, G. Andry, J.E. Dumont, Regulation of cyclic nucleotide and prostaglandin formation in normal human thyroid tissue and in autonomous nodules. J. Clin. Endocrinol. Metab. 50, 776-785 (1980)

14. L.G. Bazzara, M.L. Vélez, M.E. Costamagna, A.M. Cabanillas, L. Fozzatti, A.M. Lucero, C.G. Pellizas, A.M. Masini-Repiso, Nitric oxide/cGMP signaling inhibits TSH-stimulated iodide uptake and expression of thyroid peroxidase and thyroglobulin mRNA in FRTL-5 thyroid cells. Thyroid 17(8), 717-727 (2007)

15. H. Wang, Z. Yan, S. Yang, J. Cai, H. Robinson, H. Ke, Kinetic and structural studies of phosphodiesterase-8A and implication on the inhibitor selectivity. Biochemistry 47, 12760-12768 (2008)

16. V. Lakics, E.H. Karran, Boess,FG.: Quantitative comparison of phosphodiesterase mRNA distribution in human brain and peripheral tissues. Neuropharmacology 59, 367-374 (2010)

17. L. Persani, A. Lania, L. Alberti, R. Romoli, G. Mantovani, S. Filetti, A. Spada, M. Conti, Induction of specific phosphodiesterase isoforms by constitutive activation of the cAMP pathway in autonomous thyroid adenomas. J. Clin. Endocrinol. Metab. 85(8), 2872-2878 (2000)

18. D.S. Cooper, G.M. Doherty, B.R. Haugen, R.T. Kloos, S.L. Lee, S.J. Mandel, E.L. Mazzaferri, B. McIver, F. Pacini, M. Schlumberger, S.I. Sherman, D.L. Steward, R.M. Tuttle, Revised American Thyroid Association management guidelines for patients with thyroid nodules and differentiated thyroid cancer. Thyroid 19, 1167-1214 (2009)

19. C. Durante, G. Tallini, E. Puxeddu, M. Sponziello, S. Moretti, C. Ligorio, A. Cavaliere, K.J. Rhoden, A. Verrienti, M. Maranghi, L. Giacomelli, D. Russo, S. Filetti, BRAF(V600E) mutation and expression of proangiogenic molecular markers in papillary thyroid carcinomas. Eur. J. Endocrinol. 165(3), 455-463 (2011)

20. M.L. Sponziello, E. Lavarone, E. Pegolo, C. Di Loreto, C. Puppin, M.A. Russo, R. Bruno, S. Filetti, C. Durante, D. Russo, A. Di Cristofano, G. Damante, Molecular differences between human thyroid follicular adenoma and carcinoma revealed by analysis of a murine model of thyroid cancer. Endocrinology 154, 3043-3053 (2013)

21. M.L. Sponziello, R. Bruno, C. Durante, M. D’Agostino, R. Corradino, P. Giannasio, E. Ciociola, E. Ferretti, M. Maranghi, A. Verrienti, G. De Toma, S. Filetti, D. Russo, Growth factor receptors gene expression and Akt phosphorylation in benign human thyroid nodules are unaffected by chronic thyrotropin suppression. Horm. Metab. Res. 43(1), 22-25 (2011)

22. R.E. Schweppe, J.P. Klopper, C. Korch, U. Puqazhenthi, M. Benezra, J.A. Knauf, J.A. Fagin, L.A. Marlow, J.A. Copland, R.C. Smallridge, B.R. Haugen, Deoxyribonucleic acid profiling analysis of 40 human thyroid cancer cell lines reveals crosscontamination resulting in cell line redundancy and misidentification. J. Clin. Endocrinol. Metab. 93(11), 4331-4341 (2008)

23. S. Bulotta, R. Corradino, M. Celano, J. Maiuolo, M. D'Agostino, M. Oliverio, A. Procopio, S. Filetti, D. Russo, Antioxidant and antigrowth action of peracetylated oleuropein in thyroid cancer cells. J. Mol. Endocrinol. 51, 181-189 (2013)

24. M. D’Agostino, P. Voce, M. Celano, M. Sponziello, S. Moretti, V. Maggisano, A. Verrienti, C. Durante, S. Filetti, E. Puxeddu, D. Russo, Sunitinib exerts only limited effects on the proliferation 
and differentiation of anaplastic thyroid cancer cells. Thyroid 22, 138-144 (2012)

25. V. Maggisano, C. Puppin, M. Celano, M. D'Agostino, M. Sponziello, S. Micali, M. Navarra, G. Damante, S. Filetti, D. Russo, Cooperation of histone deacetylase inhibitors SAHA and valproic acid in promoting sodium/iodide symporter expression and function in rat Leydig testicular carcinoma cells. Endocrine 45(1), 148-152 (2014)

26. S. Bulotta, M.V. Ierardi, J. Maiuolo, M.G. Cattaneo, A. Cerullo, L.M. Vicentini, N. Borgese, Basal nitric oxide release attenuates cell migration of HeLa and endothelial cells. Biochem. Biophys. Res. Commun. 386(4), 744-749 (2009)

27. F. Arturi, D. Russo, D. Giuffrida, M. Schlumberger, S. Filetti, Sodium-iodide symporter (NIS) gene expression in lymph-node metastases of papillary thyroid carcinomas. Eur. J. Endocrinol. 143(5), 623-627 (2000)

28. D. Russo, G. Damante, E. Puxeddu, C. Durante, S. Filetti, Epigenetics of thyroid cancer and novel therapeutic targets. J. Mol. Endocrinol. 46(3), R73-R81 (2011)

29. D. Vu-Phan, R.J. Koenig, Genetics and epigenetics of sporadic thyroid cancer. Mol. Cell. Endocrinol. 386(1-2), 55-66 (2014)

30. A.L. Galrão, A.K. Sodré, R.Y. Camargo, C.U. Friguglietti, M.A. Kulcsar, E.U. Lima, G. Medeiros-Neto, I.G. Rubio, Methylation levels of sodium-iodide symporter (NIS) promoter in benign and malignant thyroid tumors with reduced NIS expression. Endocrine 43(1), 225-229 (2013)

31. C. Puppin, F. D’Aurizio, A.V. D'Elia, L. Cesaratto, G. Tell, D. Russo, S. Filetti, E. Ferretti, E. Tosi, T. Mattei, A. Pianta, L. Pellizzari, G. Damante, Effects of histone acetylation on sodium iodide symporter promoter and expression of thyroid-specific transcription factors. Endocrinology 146(9), 3967-3974 (2005)
32. E. Puxeddu, C. Durante, N. Avenia, S. Filetti, D. Russo, Clinical implication of BRAF mutation in thyroid carcinoma. Trends Endocrinol. Metab. 19, 138-145 (2008)

33. L.M. Caronia, J.E. Phay, M.H. Shah, Role of BRAF in thyroid oncogenesis. Clin. Cancer Res. 17(24), 7511-7517 (2011)

34. T. Kunavisarut, Diagnostic biomarkers of differentiated thyroid cancer. Endocrine 44(3), 616-622 (2013)

35. E. Puxeddu, S. Filetti, BRAF mutation assessment in papillary thyroid cancer: are we ready to use it in clinical practice? Endocrine 45(3), 341-343 (2014)

36. T. Keravis, C. Lugnier, Cyclic nucleotide phosphodiesterase (PDE) isozymes as targets of the intracellular signalling network: benefits of PDE inhibitors in various diseases and perspectives for future therapeutic developments. Br. J. Pharmacol. 165(5), 1288-1305 (2011)

37. H.A. Ghofrani, I.H. Osterloh, F. Grimminger, Sildenafil: from angina to erectile dysfunction to pulmonary hypertension and beyond. Nat. Rev. Drug Disc. 5, 689-702 (2006)

38. S.L. Archer, E.D. Michelakis, Phosphodiesterase type 5 inhibitors for pulmonary arterial hypertension. N. Engl. J. Med. 361(19), 1864-1871 (2009)

39. G. Corona, N. Mondaini, A. Ungar, E. Razzoli, A. Rossi, F. Fusco, Phosphodiesterase type 5 (PDE5) inhibitors in erectile dysfunction: the proper drug for the proper patient. J. Sex Med. 8(12), 3418-3432 (2011)

40. S.H. Francis, J.D. Corbin, PDE5 inhibitors: targeting erectile dysfunction in diabetics. Curr. Opin. Pharmacol. 11, 683-688 (2011)

41. D.H. Maurice, H. Ke, F. Ahmad, Y. Wang, J. Chung, V.C. Manganiello, Advances in targeting cyclic nucleotide phosphodiesterases. Nat. Rev. Drug Discov. 13(4), 290-314 (2014) 